\title{
A vertebrate-conserved cis-regulatory module for targeted expression in the main hypothalamic regulatory region for the stress response
}

\author{
Jose Arturo Gutierrez-Triana ${ }^{1,2}$, Ulrich Herget ${ }^{1,3}$, Patrick Lichtner ${ }^{1}$, Luis A Castillo-Ramírez ${ }^{1,3}$ and Soojin Ryu ${ }^{\text {** }}$
}

\begin{abstract}
Background: The homeodomain transcription factor orthopedia (Otp) is an evolutionarily conserved regulator of neuronal fates. In vertebrates, Otp is necessary for the proper development of different regions of the brain and is required in the diencephalon to specify several hypothalamic cell types, including the cells that control the stress response. To understand how this widely expressed transcription factor accomplishes hypothalamus-specific functions, we performed a comprehensive screening of otp cis-regulatory regions in zebrafish.
\end{abstract}

Results: Here, we report the identification of an evolutionarily conserved vertebrate enhancer module with activity in a restricted area of the forebrain, which includes the region of the hypothalamus that controls the stress response. This region includes neurosecretory cells producing Corticotropin-releasing hormone (Crh), Oxytocin (Oxt) and Arginine vasopressin (Avp), which are key components of the stress axis. Lastly, expression of the bacterial nitroreductase gene under this specific enhancer allowed pharmacological attenuation of the stress response in zebrafish larvae.

Conclusion: Vertebrates share many cellular and molecular components of the stress response and our work identified a striking conservation at the cis-regulatory level of a key hypothalamic developmental gene. In addition, this enhancer provides a useful tool to manipulate and visualize stress-regulatory hypothalamic cells in vivo with the long-term goal of understanding the ontogeny of the stress axis in vertebrates.

Keywords: Otp, Evolutionarily conserved non-coding regions, cis-regulatory module, Hypothalamic-pituitary-adrenal axis, Neurosecretory preoptic area, Cortisol, Zebrafish, Stress response

\section{Background}

The homeodomain protein Orthopedia (Otp) belongs to an ancient family of transcription factors involved in cell fate specification in the central nervous system of invertebrates and vertebrates [1-3].

In vertebrates, Otp is required for the proper development of the neuroendocrine hypothalamus. Genetic analyses in mice, and more recently in zebrafish, have shown that Otp, together with the heterodimeric complex formed by the bHLH-PAS transcription factors Sim1 and Arnt2, is required for the development of virtually all neuroendocrine cells in the hypothalamus. When Otp and Sim1 functions are eliminated due to a genetic mutation or morpholino injection, the cells

\footnotetext{
* Correspondence: soojin.ryu@mpimf-heidelberg.mpg.de

${ }^{1}$ Developmental Genetics of the Nervous System, Max Planck Institute for

Medical Research, Jahnstrasse 29, D-69120 Heidelberg, Germany

Full list of author information is available at the end of the article
}

expressing Arginine vasopressin (Avp), Oxytocin (Oxt), Corticotropin-releasing hormone (Crh), Thyrotropinreleasing hormone (Trh) or Somatostatin (Ss) fail to develop [3-11]. Interestingly, in the protostome polychaete Platynereis dumerilli, Otp is expressed in the medial neurosecretory forebrain region and overlaps with vasotocinergic and RFamidergic neurons [12]. These results indicate an evolutionarily conserved role of Otp for the specification of the neurosecretory control centers in bilaterian animals [13].

The hypothalamic neurosecretory control center is a component of the hypothalamic-pituitary-adrenal or hypothalamic-pituitary-interrenal axis (HPA axis or HPI axis in fish), which carry out, among other functions, a series of integrated and hierarchical physiological responses mediating the interaction of an animal with the environment under stress [14]. This system is considered to be a vertebrate innovation, such that many cellular and 
molecular components are found in cyclostomes and gnathostomes but not in cephalochordates and urochordates [15-17]. Exogenous and endogenous environmental factors can activate the HPA axis and stimulate specific neurosecretory cells in the hypothalamus to secrete hormones such as Crh, Avp and Oxt. These hormones reach the pituitary and trigger the release of adrenocorticotropic hormone $(\mathrm{ACTH})$, which then stimulates the production of glucocorticoids (GCs) in the adrenal or interrenal gland $[18,19]$. Downstream effects are then mediated by the action of GCs in peripheral organs as well as their central targets $[20,21]$.

Although the most well-known function of Otp is within the neuroendocrine hypothalamus, Otp is also expressed and involved in the specification of different cell types in multiple regions of the brain [22,23]. For example, just within the hypothalamus, Otp expression can be found in the retrochiasmatic, ventral tuberal and mammillary areas in the mouse and in the posterior hypothalamus in the zebrafish $[2,23,24]$. Furthermore, in the posterior hypothalamus of the zebrafish, Otp plays a crucial role in the specification of vasoactive intestinal peptide (VIP)-producing cells [23]. Its broad expression and involvement in the specification of different cell types raises the question as to how Otp achieves regional and cell-type specific expression and functions. In particular, we asked how Otp expression and function in the neuroendocrine hypothalamus is mediated in contrast to other regions. To address this question, we chose to analyze the regulatory landscape influencing the spatial expression of the otp gene. Specifically, we used zebrafish as a model system to identify cis-regulatory modules that drive region-specific expression of the otp gene particularly in the neuroendocrine hypothalamus that controls the stress response. The zebrafish is a very tractable organism for experimental regulatory genomics due to the availability of transgenic tools that allow visualization of fluorescent proteins reporting the regulatory potential of discrete genomic elements in a transparent embryo [25,26]. Further, the zebrafish is a promising model organism for stress research [27]. The hypothalamic region that controls the stress response is found in the paraventricular nucleus (PVN) in mammals. Its homologous region is found in the neurosecretory preoptic area (NPO) in the larval zebrafish [28]. While previous studies have analyzed cis-regulatory regions of the otp gene in zebrafish, none of the identified regions were sufficient to restrict otp expression to the NPO $[29,30]$.

Vertebrate genomes share highly conserved non-coding sequences mainly clustered close to genes involved in embryonic development and likely involved in the genomic circuitry of these genes [31]. Therefore, in the present work, we screened highly conserved non-coding regions of the Otp gene of vertebrates and identified a regulatory element with activity in a restricted area of the forebrain. Among the cells labeled by this enhancer, we found the neurosecretory cells of the HPI axis that produce $\mathrm{Crh}$, Oxt or Avp but not the diencephalic dopaminergic (DA) cells. When combined with a genetic ablation technique, this regulatory element allowed attenuation of the stress response. Taken together, these data suggest that a modular evolutionarily constrained regulatory code has been used by the gene Otp in gnathostomes to specify cells in the neurosecretory hypothalamus.

\section{Results}

The vertebrate Otp gene is flanked by evolutionarily conserved non-coding sequences

To identify and characterize functional cis-regulatory elements of the otp gene, we used a phylogenetic footprinting approach [25,32] to screen for evolutionarily conserved non-coding sequences (ECRs) in or around the gene using the ECR browser (http://ecrbrowser.dcode.org) and compared the genomic sequences flanking the otp gene of human, mouse and zebrafish (otpa). To determine whether these ECRs are conserved in different vertebrates, we performed individual pairwise BLASTn comparisons using the standard nucleotide Blast [33] optimized for somewhat similar sequences using the genome databases of the $\mathrm{Na}$ tional Library of Medicine (http://blast.ncbi.nlm.nih.gov), Ensembl 2014 (http://www.ensembl.org [34]) and the available genome sequences of the sarcopterygian Coelacanth (Latimeria chalumnae), the chondrichthyan elephant shark (Challorhincus milii), and agnathan lampreys (Lethenteron japonicum and Petromyzon marinus) (http://www.ensembl. org/Latimeria_chalumnae; http://esharkgenome.imcb.astar.edu.sg [35]; http://jlampreygenome.imcb.a-star.edu.sg [36]). The minimum length of the regions was set to $80 \mathrm{bp}$, and the identity was set to above $70 \%$.

Several ECRs with more than $70 \%$ identity were identified, including 6 ECRs in the $5^{\prime}$ upstream region and 1 in the 3 ' downstream region (Figure 1A). Interestingly, all of the $5^{\prime}$ upstream ECRs, except for otpECR3, are present in the upstream region of the otp gene in gnathostome vertebrates. The overall percentage of identity is remarkably high $(>92 \%$ for otpECR5, $>85 \%$ for otpECR4, $>83 \%$ for otpECR2, $>80 \%$ for otpECR6, $>78 \%$ for otpECR1, and $>72 \%$ for $o t p E C R 3$, see Additional file 1: Figure S1). In the pairwise comparisons of human and zebrafish, the values are similar to the global comparison (92\% for otpECR5, $88 \%$ for otpECR4 and otpECR2, 86\% for otpECR6, and $78 \%$ for $o t p E C R 3$ and $o t p E C R 1)$. Interestingly, in the zebrafish paralogous otp gene, otpb, ECR3, ECR4, and ECR5 are absent. Furthermore, the percentage of identity of the remaining otpb ECRs is lower than the identity of the orthologous blocks in the vertebrates analyzed (e.g., $>59 \%$ for $o t p E C R 6)$. Although an otp gene is found in the genome of the lamprey, none of the ECRs were identified 

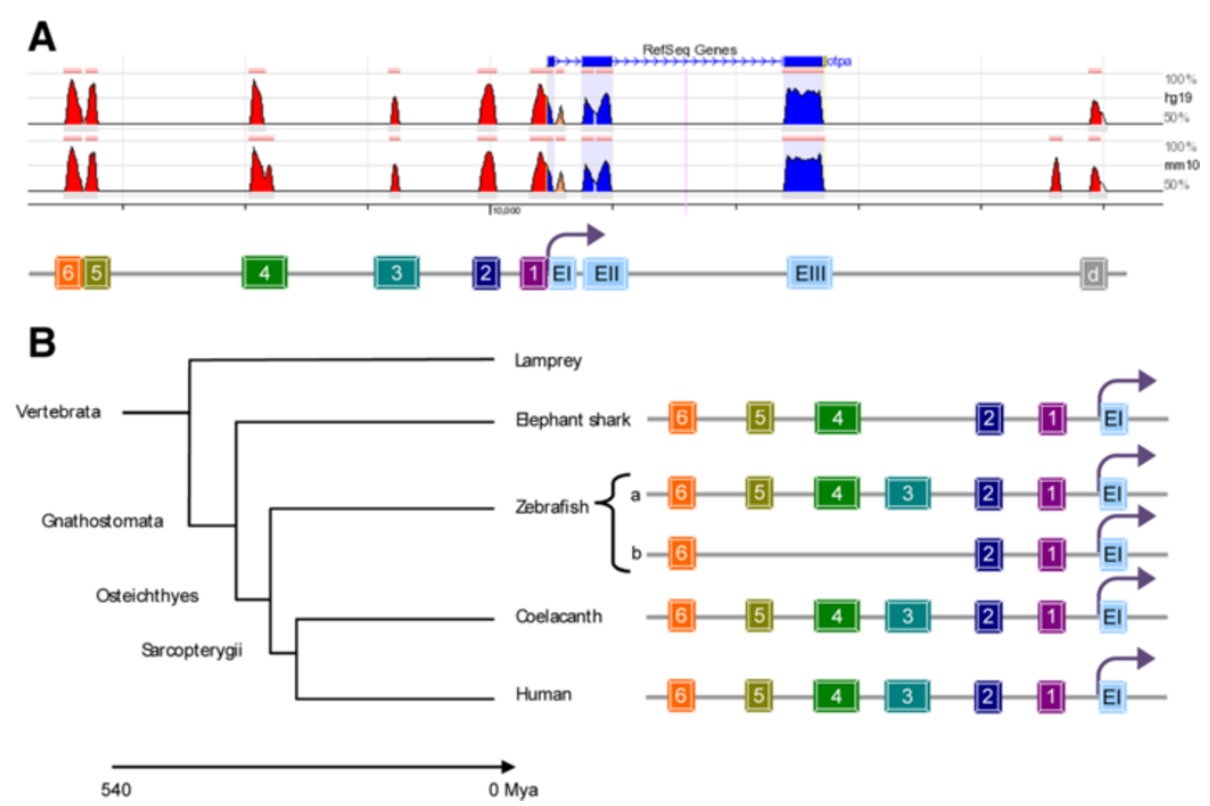

Figure 1 Genomic structure and evolutionarily conserved non-coding regions (ECRs) of the otp gene. A, Graphical comparison of the otp locus, according to the ECR browser, showing intergenic ECRs (red) and exons (blue) in the zebrafish (genome assembly danRer7), the human (genome assembly hg19) and the mouse (genome assembly mm10). We used the sequence of the otpa gene from zebrafish as the reference. B, A schematic phylogenetic tree of vertebrates. otp ECRs are evident only in gnathostome vertebrates. We noted that some ECRs were absent in the paralogous gene otpb in zebrafish (see text for more details).

under the parameters used for the analysis (Figure 1B), suggesting an innovation in the regulatory network controlling this gene in jawed vertebrates.

\section{otpECR6 is a cis-regulatory module driving expression in the NPO of the zebrafish brain}

To evaluate the regulatory activity of the ECRs identified in the vertebrate Otp genes, we analyzed the expression of individual ECRs in transgenic zebrafish using GFP as a reporter. To do this, individual ECRs around the otpa gene, from 2 to 6 , were cloned into a modified version of the ZED vector (a Tol2-based plasmid carrying insulator sequences to decrease variability in the GFP expression pattern due to integration site effects [37]). The plasmids also contained a cassette expressing RFP under a cardiac promoter as a transgenesis marker. These plasmids were injected into zebrafish embryos and analyzed at 24 hours post-fertilization (hpf), $72 \mathrm{hpf}$, and 5 days post-fertilization (dpf) in both transient assays and using stable transgenic fish lines. At least three independent stable transgenic lines were established per plasmid. In our analyses, we excluded ECR1, which contains the TATA box, because this region performed as a basal promoter that could be used to trap neighboring enhancers. For instance, from our initial study, one of the transgenic zebrafish lines using the otpECR1 showed GFP expression specifically in the habenula, although otpa is normally not expressed there [38]. Notably, we failed to detect GFP expression in all ECRs except $o t p E C R 6$ in transgenic lines that were identified using the RFP expression in the heart (Figure 2B). otpECR6 drives a robust forebrain expression even in transientinjected animals (data not shown). Further characterization was performed using the stable line $\operatorname{Tg}$ (otpECR6-E1b: $m m G F P$ ). In anatomical terms, this region corresponds primarily to the zebrafish neurosecretory preoptic area (NPO) and the dorso-rostral extension of the otpa domain in the caudal telencephalon [28]. We also observed ectopic and mosaic GFP expression in a small group of cells in a dorsal region of the posterior midbrain of larvae (Figure 2C,D). This mosaicism probably reflects multiple insertions of the transgene in the founder genome, as is reported for Tol2mediated transgenesis in zebrafish [39].

The conserved DNA sequence of otpECR6 contains predicted binding sites for transcription factors belonging to the families Otx, Pax, Nkx and Zic (Figure 2E). To test for regulatory interactions of members of any of these TF families with the otpECR6 module, we co-injected, into one-cell stage embryos, the reporter plasmid otpECR6E1b:mmGFP, which contains otpECR6 fused to the E1b basal promoter and the fluorophore mmGFP, together with mRNA encoding a chimeric protein consisting of a candidate transcription factor fused to the strong activation domain of the VP16 protein [40]. We then checked for GFP expression at 6-10 hours post fertilization (hpf). This transcriptional activation assay was performed using 


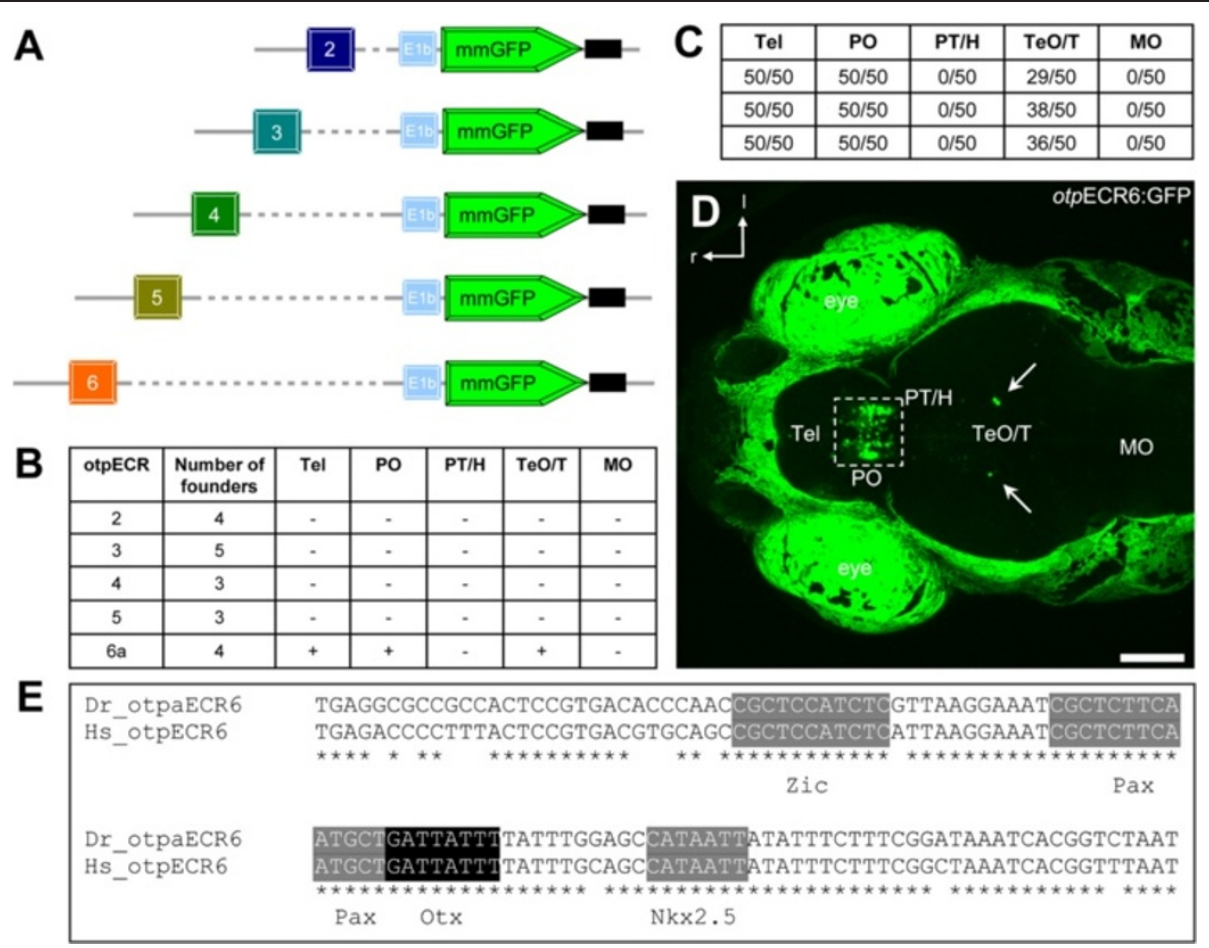

Figure 2 Enhancer activity displayed by stable otpECR-transgenic lines. A, A schematic depiction of constructs used to generate the transgenic lines. B, Enhancer activity displayed by transgenic lines analyzed at 3-5 dpf. The table shows the GFP levels and expression patterns observed in cardiac-RFP positive founders for the individual otpECRs. C, Numbers of larvae showing GFP+ cells within different brain regions (Data from 3 independent clutches are shown, $n=50$ per clutch). In all animals, the caudal telecephalic-preoptic cluster is present, and in many animals, few ectopic cells appear in the tectal-tegmental region. D, Confocal z-stack maximum projection of a dorsal in vivo view of Tg(otpECR6-E1b:mmGFP) expression. GFP is expressed in a dense cluster within the preoptic area and extends into the caudal telencephalon. In many larvae, a few isolated ectopic cells can be detected in the midbrain. $\mathbf{E}$, The evolutionarily conserved region otpECR6 contains predicted binding sites for transcription factors belonging to the families Otx, Pax, Nkx and Zic. The otpECR6 DNA sequences of zebrafish (Dr) and human (Hs) were aligned and analyzed using the multiF program (https://multitf.dcode.org of the ECR browser). Abbreviations: Tel, telencephalon; PO, preoptic area; PT, posterior tuberculum; $\mathrm{H}$, hypothalamus; TeO, optic tectum; T, tegmentum; MO, medulla oblongata; r, rostral; I, lateral. Scale bar: 100 um.

the zebrafish transcription factors Otx1, Otx2a, Pax2a, Pax6a, Nkx2.1a, Nkx2.1b and Zic2a, which are expressed in the hypothalamus (www.zfin.org). None of these transcription factors activated the expression of GFP (data not shown).

In the $T g$ (otpECR6-E1b:mmGFP) line, GFP-expressing cells appear starting at approximately $28-30 \mathrm{hpf}$ and are mostly found in the forebrain. At $5 \mathrm{dpf}, 91 \%$ of the GFP cells are otpa-positive, as shown by immunostaining (Figure 3). Therefore, we conclude that otpECR6 is a cisregulatory element driving modular expression mainly in a subset of otpa-expressing cells in the forebrain of zebrafish.

\section{Chemoarchitecture defined by otpECR6}

To characterize the cell identity of the GFP-expressing cells driven by otpECR6, we performed immunohistochemistry and fluorescent in situ hybridization (FISH) using probes and antibodies against different neuropeptidergic markers previously described to be present in this region [28]. GFP cells in the NPO colocalized with neurosecretory cells expressing crh, avp, oxt, or proenkephalin a (penka) (Figure 4). $89 \%$ of the oxt-positive cells, and $81 \%$ of the $a v p$-positive cells (based on IHC) showed colocalization with GFP; while $62 \%$ of the crhpositive cells, and $22 \%$ of the penka-positive cells (based on FISH) showed colocalization with GFP. In contrast, diencephalic DA cells and their projections were not included in the GFP expression domain (Figure 4). There was no colocalization with cells producing cholecystokinin (cck), vip, or proenkephalin b (penkb), and low colocalization with cells producing somatostatin (sst1.1) or neurotensin (nts). Because $c c k$, vip, penkb, and $n t$ s cluster at the border of the NPO, we concluded that otpECR6 expression overlaps more with the central core region of the NPO, where crh, avp, oxt, and penka are clustered. A morphological hallmark of hypothalamic neurosecretory cells is their projection to the pituitary. To confirm the neurosecretory nature of the cells targeted using otpECR6, we generated a transgenic line expressing a membranetagged RFP to visualize cell projections. Indeed, we observed two sets of bilateral projections in $\operatorname{Tg}$ (otpECR6-E1b: 

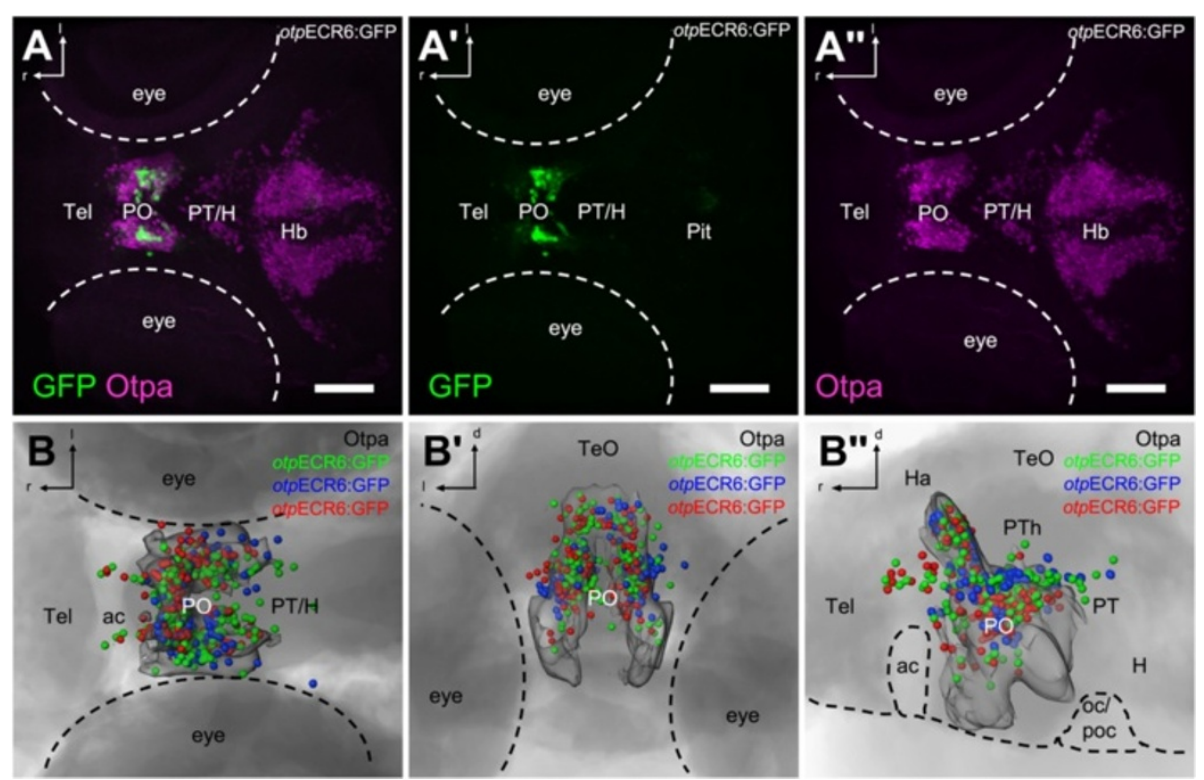

Figure 3 otpECR6 displays enhancer activity in the forebrain with expression in cells localizing within the caudal telencephalon and dorsal half of the preoptic Otpa domain. A, Confocal z-stack maximum projection of an immunohistochemically stained $5 \mathrm{dpf}$ Tg(otpECR6-E1b: mmGFP) larva double-labeled for GFP (A') and Otpa (A"). B, Reconstructed localization and variability of GFP-expressing cells. Colors represent three representative maps derived from three

different animals after registration using the outlines of the Otpa signal as references (transparent surface). Abbreviations: Tel, telencephalon; PO, preoptic area; PT, posterior tuberculum; H, hypothalamus; Hb, hindbrain; Pit, pituitary; ac, anterior commissure; TeO, optic tectum; Ha, habenula; PTh, prethalamus; oc, optic chiasm; poc, postoptic commissure; r, rostral; l, lateral; d, dorsal. Scale bars: $100 \mu \mathrm{m}$.

RFP-CAAX). One set projects caudally, ending halfway along the spinal cord (not shown), whereas the other projects to the pituitary, as shown by co-staining with an ACTH antibody (Figure 5). The RFP projections toward the pituitary colocalize with Oxt and Avp projections. Taken together, our results indicate that otpECR6 is a functional regulatory element with activity in key neurosecretory cells of the NPO.

\section{Genetic cell ablation using otpECR6 impairs the stress response}

The conditional nitroreductase-metronidazole (NM) system has been recently used in zebrafish to determine the function of a specific group of cells in a developing organism $[41,42]$. Because the otpECR6 regulatory element drives expression in neurosecretory cells involved in the HPI axis response, we hypothesized that expressing the nitroreductase protein under this regulatory element will impair the stress response when the larvae are incubated in metronidazole (Mtz). To test this directly, we generated stable transgenic zebrafish lines expressing the $E$. coli nitroreductase $n f s B$ as a GFP fusion protein under the otpECR6. To test the efficiency of the NM method, we tested the ablation efficiency of $n f s B$-GFP cells in $T g$ (otpECR6-E1b:nfsb-GFP) larvae exposed to Mtz using a TUNEL assay. At $6 \mathrm{dpf}$, we detected TUNEL-positive cells in the NPO area only in those $T g$ (otpECR6-E1b:nfsb-GFP) larvae treated with Mtz (Figure 6A, B). In addition, we observed a significant decrease in the number of projections to the pituitary in Mtz-treated embryos compared with untreated embryos (Figure 6C, D).

To test whether Mtz-induced impairment of neurosecretory NPO cells affects the stress response, we treated Tg(otpECR6-E1b:mmGFP) and Tg(otpECR6-E1b:nfsb-GFP) larvae with E2 medium alone or E2 medium supplemented with Mtz and then subjected them to a mechanical stress paradigm that has been shown to induce an intensity-dependent increase in whole-body cortisol levels in zebrafish larvae (Castillo-Ramirez, L., unpublished data). Neither genotype nor Mtz treatment affected basal cortisol levels (Figure 7B; Two way ANOVA, genotype factor: $\mathrm{F}_{(1,20)}=0.57, \mathrm{p}=0.46$, Mtz factor: $\mathrm{F}_{(1,20)}$ $=2.69, \mathrm{p}=0.12$, genotype $\mathrm{X}$ Mtz factor: $\mathrm{F}_{(1,20)}=0.65, \mathrm{p}=$ 0.43). Stressor exposure increased cortisol level in both $\mathrm{Tg}$ (otpECR6-E1b:nfsb-GFP) and Tg(otpECR6-E1b:mmGFP) larvae, regardless of Mtz incubation (Figure 7C; One sample t-test against 0 , GFP -Mtz: $\mathrm{t}_{(5)}=16.6, \mathrm{p}<0.0001$, GFP + Mtz: $t_{(5)}=7.1, p=0.0009$, nfsb-GFP - Mtz: $t_{(5)}=$ 18.4, $\left.\mathrm{p}<0.0001, \quad \mathrm{nfsb}-\mathrm{GFP}+\mathrm{Mtz}_{\mathrm{t}} \mathrm{t}_{(5)}=10.7, \mathrm{p}<0.0001\right)$. However, although Mtz treatment reduced stressorinduced cortisol increase in both groups, the reduction was much greater in $T g$ (otpECR6-E1b:nfsb-GFP) larvae (Figure 7C; Two way ANOVA, genotype factor: $\mathrm{F}_{(1,20)}=$ 8.90, $\mathrm{p}=0.007$, Mtz factor: $\mathrm{F}_{(1,20)}=130.1, \mathrm{p}<0.0001$, 


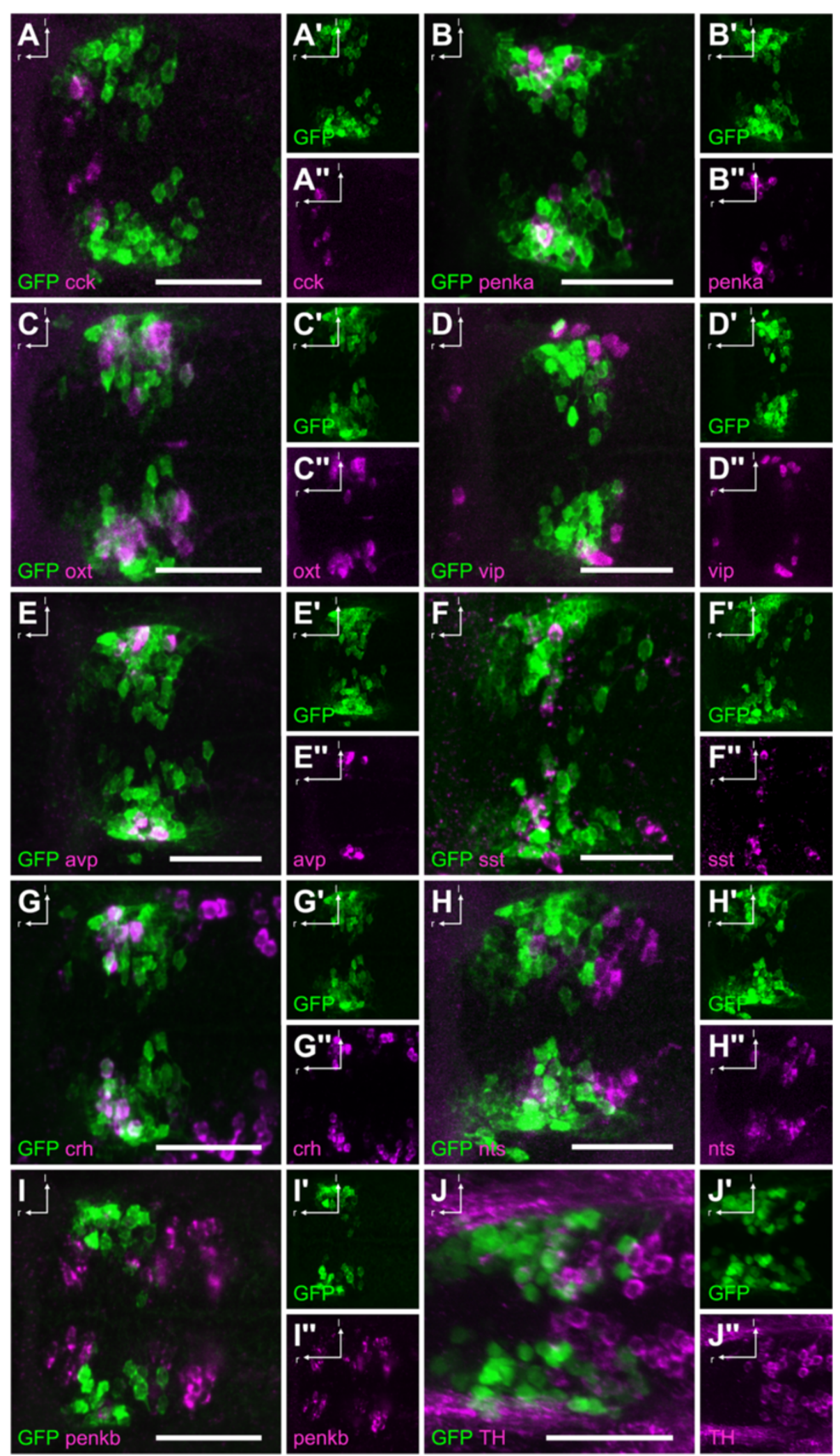

Figure 4 (See legend on next page.) 
(See figure on previous page.)

Figure 4 NPO cells involved in the HPA axis are labeled in the Tg(otpECR6-E1b:mmGFP) transgenic line. A, There is no overlap of GFP with in situ stained cck+ cells. B, There is some overlap of GFP with penka+ cells. C, There is extensive overlap of GFP with oxt+ cells. D, There is no overlap of GFP with vip+ cells. $\mathbf{E}$, There is a high degree of overlap of GFP with avp+ cells. F, There is some overlap of GFP with sst 1.1+ cells. G, There is a high degree of overlap of GFP with crh + cells. $\mathbf{H}$, There is some overlap of GFP with nts + cells. I, There is no overlap of GFP with penkb + cells. J, There is no overlap of GFP with immunostained TH+ cells. Abbreviations: $r$, rostral; I, lateral. Scale bars: $50 \mu \mathrm{m}$.

genotype X Mtz factor: $\left.\mathrm{F}_{(1,20)}=5.54, \mathrm{p}=0.03\right)$. In fact, cortisol increase differed between the two genotypes only in the presence of Mtz (Figure 7C; Bonferroni post hoc test, GFP -Mtz vs. nfsb-GFP -Mtz, p > 0.05; GFP + Mtz vs. nfsb-GFP + Mtz, $\mathrm{p}<0.01$ ). This provides evidence for the Mtz-induced impairment of the endocrine response in $\operatorname{Tg}$ (otpECR6-E1b:nfsb-GFP) larvae supporting the use of otpECR6 for functional manipulation of the HPI axis.

\section{Discussion}

Here, we report the identification and functional characterization of a cis-regulatory module (CRM) of the gene Otp with regulatory activity in the main stress-controlling hypothalamic region (NPO) in the zebrafish brain. This CRM is highly conserved in jawed vertebrates, and it restricts gene expression to a forebrain area which includes the neurosecretory cells involved in the HPI axis. Accordingly, when otpECR6 was used to pharmacologically ablate these cells, we observed a significant impairment of the stress response.

Otp is an ancient transcription factor gene with a conserved expression in the vertebrate brain. Similar to other developmental regulators, the Otp gene is flanked by highly conserved non-coding sequences. Identifying precise functions of such sequences represents one of the pressing challenges of modern genomics research. Although evolutionary conservation at the sequence level indicates selective constraints against mutations [43], the correlation of ECRs with gene regulatory functions is not straightforward $[44,45]$. We used the zebrafish as a model organism to evaluate the cis-regulatory potential of a selected group of ECRs identified through extensive comparisons of the intergenic sequences of the Otp gene in representative species of vertebrates. We defined 6 ECRs consisting of a minimum of 80 bases with more than $70 \%$ nucleotide identity in the upstream region of the gene. Interestingly, none of the ECRs were evident in the genomes of the lampreys studied, although we found a
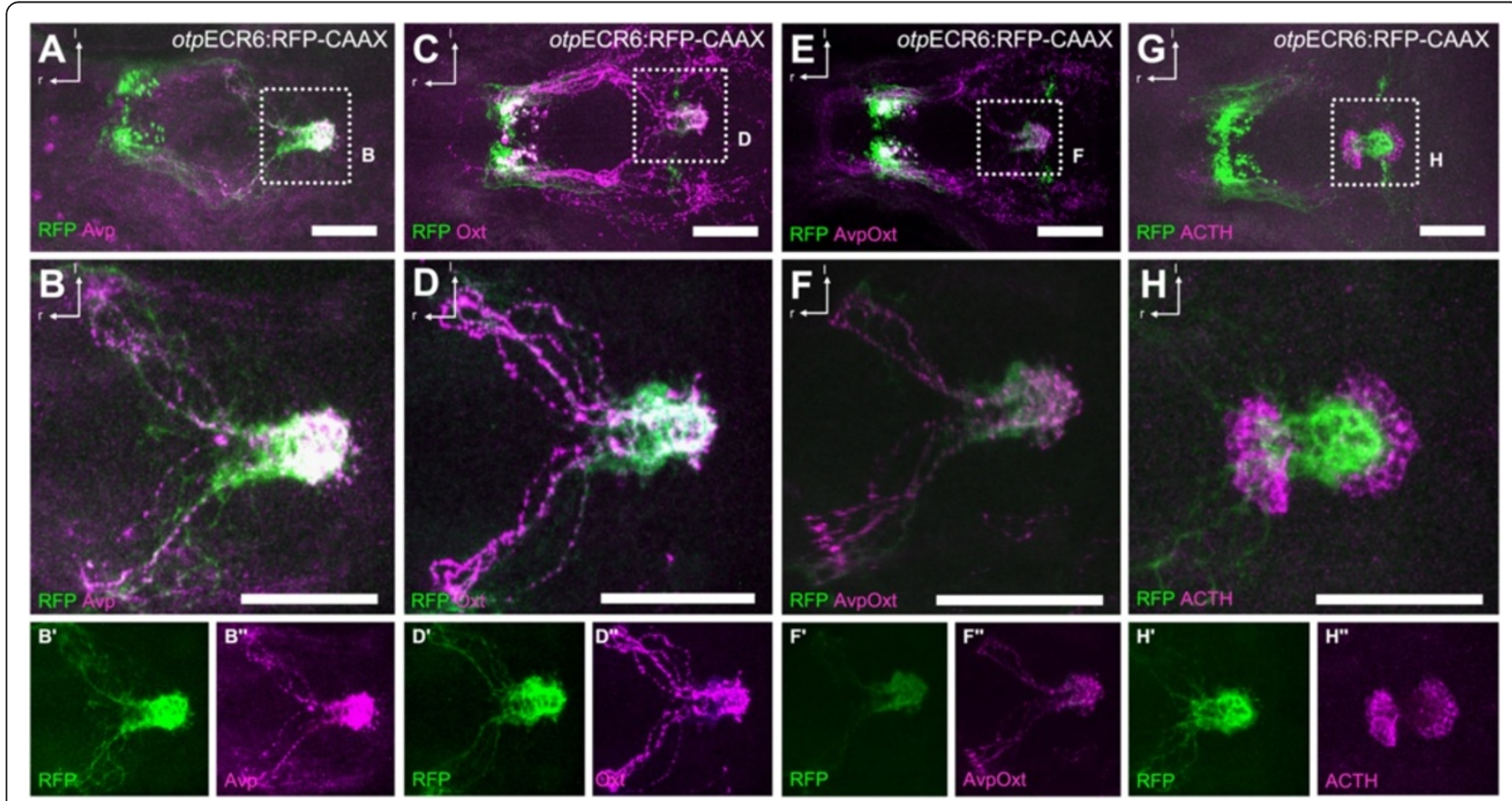

Figure 5 A group of cells labeled by the activity of the otpECR6 enhancer project to the pituitary. A-D, Costaining of RFP with Avp (A) or Oxt (C) as typical hypophysiotropic cell types shows a high degree of overlap of fibers reaching the pituitary (magnified views in $\mathbf{B}$ and $\mathbf{D}$ ). E, Costaining of RFP with Avp and Oxt combined (magnified view in F). G, Costaining with ACTH as a pituitary marker confirms the dense RFP bundles as part of the pituitary (magnified view in $\mathbf{H}$ ). Abbreviations: r, rostral; I, lateral. Scale bars: $100 \mu \mathrm{m}$. 

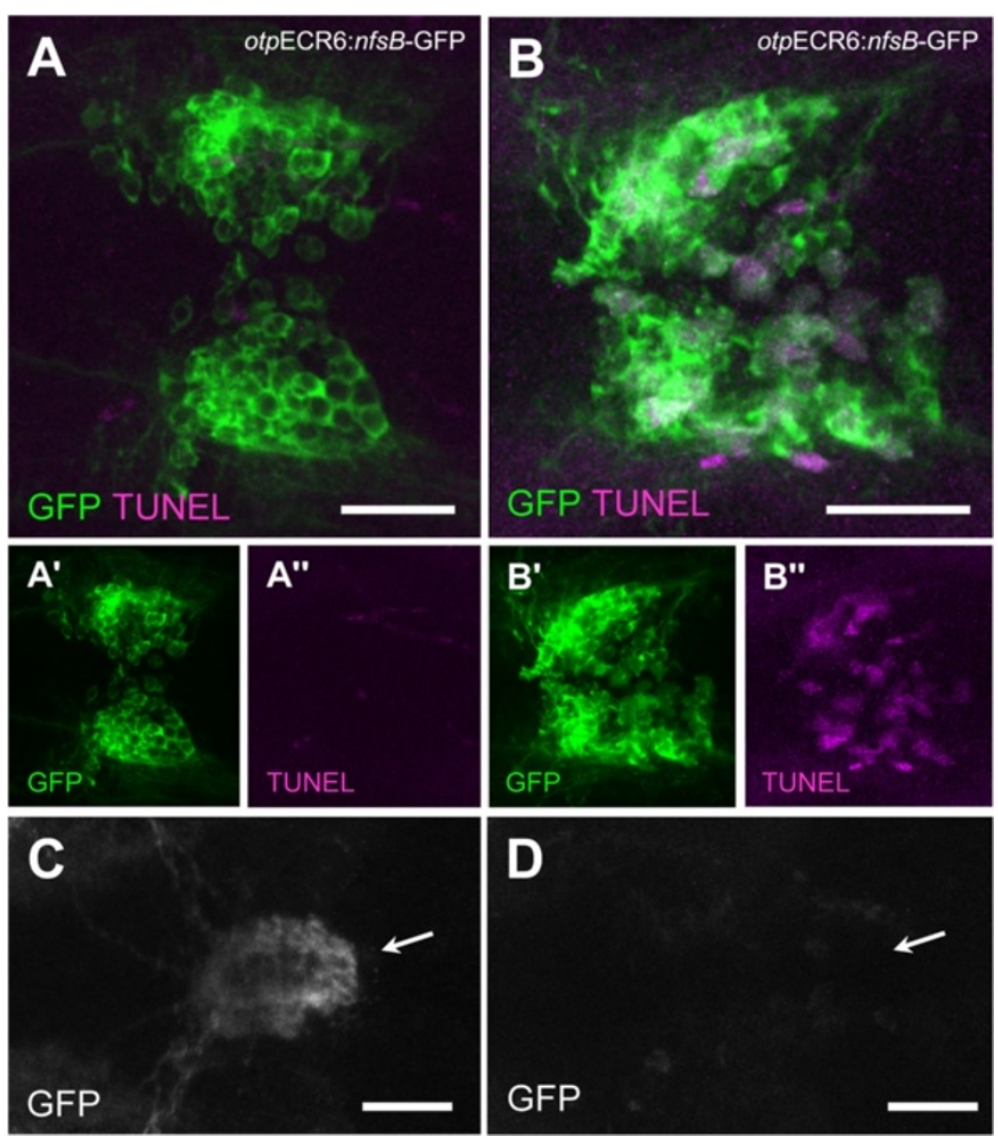

Figure 6 Nitroreductase-expressing cells in Tg(otpECR6-E1b:nfsb-GFP) larvae display a reduction in the number of projections to the pituitary and an increase in apoptotic bodies when exposed to Mtz. A, TUNEL staining of the preoptic GFP expression domain in an untreated larva. B, TUNEL staining of the preoptic GFP expression domain in an Mtz-treated larva. C, Pituitary bundles of GFP-stained fibers in a control larva (arrow). D, The pituitary location in an Mtz-treated larva (arrow). Note that in the control larva, the GFP fibers reach the pituitary and no apoptosis is detected, but in the Mtz-treated larva, the pituitary innervation is disrupted and apoptosis is apparent in preoptic cell bodies. Scale bars: $50 \mu \mathrm{m}$.

copy of the Otp gene. This could be due to intrinsic problems in the genome assembly in these organisms due to the programmed genome rearrangement occurring in certain somatic tissues during embryonic development [46]. Alternatively, the ECRs could have evolved specifically in the ancestor of gnathostomes just after the second genome duplication event that occurred in the cyclostome-gnathostome lineage [47]. It is important to note that many neuropeptide genes whose identities are specified by Otp in gnathostomes are also present in cyclostomes [15], suggesting that an ancestral genetic network mediated by Otp in the diencephalon was already established in cyclostomes.

In our approach, we evaluated the regulatory potential of individual ECRs in zebrafish larvae. As a framework, we looked for GFP reporter activity of individual ECRs in the context of the Otp expression domain. Surprisingly, only one ECR was shown to be a true CRM, the element $o t p E C R 6$. The inability to detect GFP expression with the other ECRs points to a combinatorial use of these regions to achieve a regional activity [48]. Alternatively, some ECRs are used by repressors and will not activate the reporter. Moreover, the PCR product representing some of the tested ECRs could have omitted non-conserved critical transcription factor-binding sites required to maintain the full activity of the region. To prevent this problem, ECRs were flanked by more than $100 \mathrm{bp}$.

Interestingly, the identified CRM, otpECR6, is included in the human enhancer hs262 reported in the Vista Enhancer Browser resource for conserved non-coding elements of the human genome and tested in mouse embryos (http://enhancer.lbl.gov). The enhancer hs262 displays a conspicuous lacZ activity in a restricted area of the mouse ventral forebrain, similar to the otpECR6 activity reported here. In addition, otpECR6 overlaps with a DNA track, reported in the Encyclopedia of DNA elements (ENCODE), showing significant levels of enrichment of the H3K27Ac histone mark, which is one of the characteristic signatures of an active enhancer 


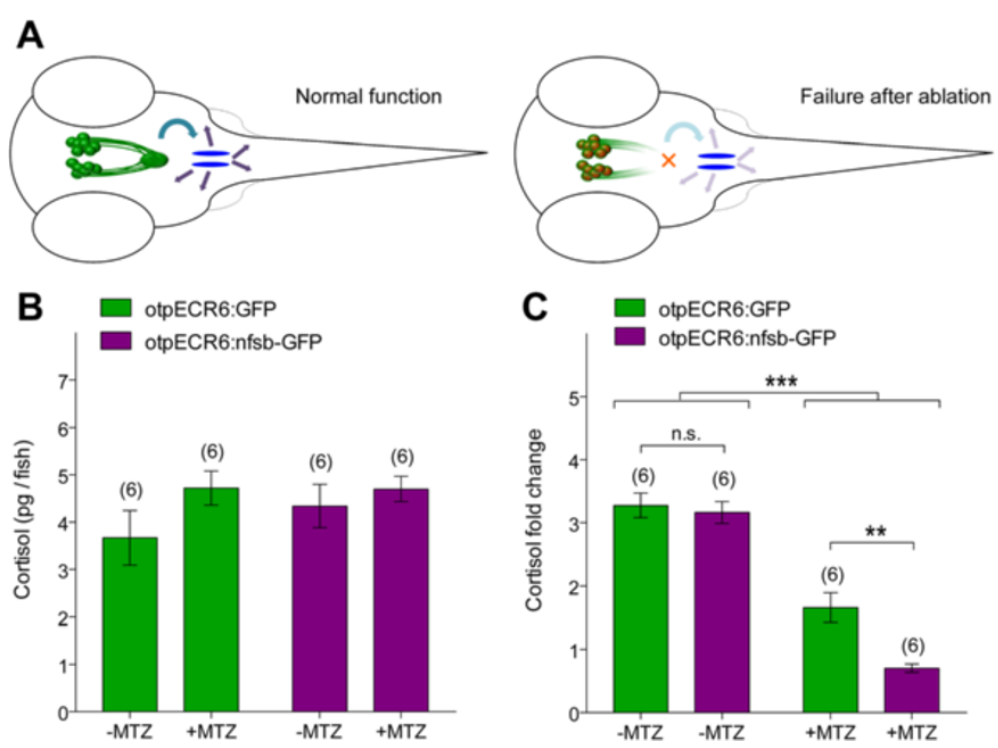

Figure 7 Mtz-induced impairment of the cortisol response to stress in Tg(otpECR6-E1b:nfsb-GFP) larvae. A, A schematic illustration of a functional stress axis (fibers of the hypothalamo-pituitary complex in green, interrenal gland in blue) and an impaired stress axis after Mtz treatment. B-C, Basal and stress-induced cortisol levels in Tg(otpECR6-E1b:mmGFP) and Tg(otpECR6-E1b:nfsb-GFP) larvae, treated or untreated with Mtz.

(ENCODE Project Consortium, 2011). Together, these data suggest that otpECR6 is an evolutionarily conserved DNA element with a conserved regulatory function in the forebrain of vertebrates.

Evolution of a gene's function may occur through modifications of the architecture of its regulatory networks $[49,50]$. A whole-genome duplication event occurred in the ancestor lineage of ray-finned fishes approximately 350 million years ago [51]. This duplication event is proposed to explain the tremendous diversification of teleosts by releasing an important set of genomic mechanisms that allowed the evolution of gene function [52]. In zebrafish, the otp gene is present in two copies, otpa and $o t p b$. We noticed that the percentage of identity of otpECR6 was higher when comparing the otpa copy with the orthologous Otp genes than when comparing the same region of the otpb copy. In addition, the ECR landscape of the otpb locus lacks ECRs 3, 4 and 5. At the amino acid level, Otpa resembles more the mammalian counterpart [11]. In a recent paper, it has been shown that Otpa and Otpb have partially redundant roles in the development of the zebrafish diencephalon; however, Otpa has a more prominent role in the specification of neuroendocrine and dopaminergic cells [22]. Furthermore, the expression domains of otpa and $o t p b$ differ in the NPO and its surrounding areas in $5 \mathrm{dpf}$ zebrafish larvae, where the otpb gene is expressed in a medial subregion within a larger otpa domain and otpa extends into the caudal telencephalon [28]. These results support the idea that the paralogous otp gene in zebrafish, otpb, has undergone a process of subfunctionalization through modifications at the cis-regulatory and amino acid levels [53].

The analysis of the chemoarchitecture defined by otpECR6 identified neurosecretory cells involved in the HPI axis. For instance, otpECR6 reporter activity colocalized with a group of cells expressing Crh, Avp, or Oxt in the neurosecretory preoptic area. Because Otp is required for the specification of neurosecretory lineages in zebrafish and mice, we can infer that the function of otpECR6 is to maintain the regional expression of the otp gene in the NPO or in the homologous region of the mammalian hypothalamus, the PVN. In a recent screen to identify DA cis-regulatory modules in genes involved in DA neuron specification or function, Fujimoto et al. [30] reported a DNA element of the otpb gene, the otpb.A enhancer, which drove expression in the diencephalic DA cells and in the Oxt cells of the NPO. otpECR6 is included within the $4.5 \mathrm{~kb}$ otpb.A enhancer. Interestingly, we did not observe any DA neurons colocalizing with GFP in otpaECR6 stable lines. This suggests that the $4.5 \mathrm{~kb}$ otpb.A enhancer is in fact a composite regulatory module containing otpECR6, which drives the expression in the NPO and unidentified regulatory sequences responsible for the expression in DA cells.

The restricted expression activity displayed by otpECR6 in the caudal telencephalon and NPO area, and in particular in the neurosecretory cells involved in the HPI axis, may be useful for functional analyses of the stress response in vertebrates. The zebrafish is a promising 
model organism to study the mechanisms underlying early stress effects on the development of adult phenotypes [27]. Many of the cellular and molecular components of the stress axis are conserved between mammals and fishes $[19,54]$, and the present work identified striking conservation at the gene regulatory level. We succeeded in altering the normal cortisol response to a mechanical stressor using conditional Mtz impairment in cells expressing the bacterial nitroreductase gene under the otpECR6 regulatory element. These results were consistent with structural changes of nitroreductase-expressing cells when exposed to Mtz (e.g., reduced projections to the pituitary). We cannot exclude potential effects on the stress response from the uncharacterized cells in the caudal telencephalon or the few ectopic dorsal midbrain cells. The otpb.A enhancer was successfully used recently in a similar chemical ablation experiment to study the role of the diencephalic DA tract (DDT) in the locomotor development of zebrafish larvae [55]. Because otpECR6 does not cover DA Otp + cells, this regulatory element can be of use to control for any effect of non-DA Otp+ cells in locomotor and behavioral experiments.

New molecular tools for neuronal activity analysis (such as GCamPs), neuronal manipulation (such as Channelrhodopsins) or single-cell morphology visualization (Brainbow) can be coupled to this regulatory element to address questions concerning the differential activation of the HPI axis under acute and chronic stress.

Our results raise further questions: 1) Is otpECR6 indispensable for the proper development of the neurosecretory hypothalamus in vertebrates? 2) If the function of otpECR6 is conserved in humans, is there any genetic variation found in this element that is associated with any stress-related condition? 3) What type of regulatory mechanisms drive otpECR6-dependent Otp expression? The answers to these questions promise to reveal new insights into the genetic networks regulating the stress axis.

\section{Conclusions}

Using a combination of comparative genomics, zebrafish transgenesis and imaging, we found that the transcription factor Otp uses a single and highly conserved cis-regulatory element, otpECR6, to achieve expression in a key neurosecretory control center in zebrafish. Our results suggest that the regulatory network used by the Otp gene in the hypothalamus was already established in the ancestor of gnathostomes. In addition, we showed that the response of the HPI axis to a mechanical stressor in zebrafish larvae is impaired when the function of the cells covered by the regulatory activity of otpECR6 is compromised using a pharmacogenetic ablation technique. Consequently, we propose this element as a tool for further functional analyses of the physiology and ontogeny of the stress response in zebrafish.

\section{Methods}

\section{Fish maintenance}

Zebrafish were bred and maintained according to standard methods [56]. Zebrafish experimental procedures were performed according to the guidelines of the German animal welfare law and approved by the ethics committee of the local government (Regional Council Karlsruhe, Germany).

\section{PCR and plasmid generation}

Individual identified evolutionarily conserved non-coding regions (ECRs) were PCR amplified from genomic DNA extracted from $3 \mathrm{dpf}$ zebrafish larvae using the primers listed in Additional file 2: Table S1. PCR products were cloned into a modified version of the pZED vector [37]. The modification consisted of the removal of the Gateway cassette, replacement of the GATA basal promoter and the EGFP with the adenovirus E1B basal promoter and the use of mmGFP [57]. The test cassette was flanked by insulator elements that reduce positional effects [58] and the plasmid contained, in antisense, a cassette expressing RFP under the cardiac myosin light chain 2 promoter as a transgenesis marker.

In addition, 3x multimerized otpECR6 was subcloned to generate different sets of plasmids: pT2-otpECR6-E1B: RFP-CAAX, where RFP-CAAX denotes a membranebound red fluorescent protein and pT2-otpECR6-E1B: $n f s b$-GFP, where $n f s b$-GFP encodes a chimeric protein consisting of the $E$. coli nitroreductase gene $n f s b 1 \mathrm{~N}$ terminally fused to GFP. In all cases, the identity of the cloned fragments was confirmed by sequencing.

\section{Plasmid injection and trangenesis}

Recombinant plasmids were injected into one-cell stage wild-type embryos at a $10 \mathrm{ng} / \mu \mathrm{l}$ concentration in the presence of $10 \mathrm{ng} / \mu \mathrm{l}$ Tol 2 transposase mRNA and $0.05 \%$ phenol red. The progenies of injected fish were maintained in E2 medium supplemented with $0.2 \mathrm{mM} \mathrm{N}$-phenylthiourea (Sigma-Aldrich, catalogue no. P7629) to prevent pigmentation. Larvae were screened for heart RFP and brain GFP expression at 2, 3, or $5 \mathrm{dpf}$ using a Leica MZ6 fluorescent dissecting microscope. At least 3 founders per construct were identified. The following transgenic lines have been established: $\operatorname{Tg}$ (otpECR6-E1b: mmGFP)hd12, Tg(otpECR6-E1b:RFP-CAAX)hd13, Tg (otpECR6-E1b:nfsb-GFP)hd14.

Immunohistochemistry and fluorescent in situ hybridization Immunohistochemistry on $3 \mathrm{dpf}$ and $5 \mathrm{dpf}$ larvae was performed as previously described [28] using the following antibodies. Primary antibodies: chicken anti-GFP (1:500, Abcam); rat anti-Otpa (1:500) [23]; rabbit anti-Avp (1:250) and rabbit anti-Oxt (1:250) [28]; rabbit anti-TH (1:250) [11]; rabbit anti-ACTH (1:1000, National Hormone and 
Peptide Program, National Institute of Diabetes and Digestive and Kidney Diseases); and rabbit anti-tagRFP (1:500, Evrogen). Secondary antibodies: goat anti-chicken Alexa Fluor 488 (1:1000, Invitrogen); goat anti-rabbit Alexa Fluor 555 (1:1000, Invitrogen); and goat anti-rat Alexa Fluor 555 (1:1000, Invitrogen).

Fluorescent in situ hybridization was performed as previously described [23] using the following in situ probes: crh, avp, oxt, sst1.1, and penka, as reported in [28]. Larvae were imaged in $80 \%$ glycerol in PBS using a Nikon 20x glycerol objective and a Leica SP5 confocal laser scanning microscope. Confocal image channels were recorded sequentially with optimal gain, offset, zoom, and dimension settings for the desired volume. Stacks were subsequently evaluated using Amira 5.4 software (FEI Visualization Sciences Group) to create maximum intensity projections, perform 3D registration, and reconstruct the spatial arrangement of cells [28]. Maximum intensity projections were restricted to the volume of interest, excluding signals from above or below. Brightness and contrast were adjusted for each channel.

\section{Conditional targeted cell-ablation}

Conditional targeted cell-ablation was performed as reported [41] using a solution of $10 \mathrm{mM} \mathrm{Mtz} \mathrm{(Sigma-Aldrich}$ catalog no. M1547) in E2 medium supplemented with $0.2 \mathrm{mM}$ N-phenylthiourea (PTU). The experimental treatments were as follows: $n f s B$-GFP-positive or $n f s B$-GFPnegative embryos were sorted at $3 \mathrm{dpf}$ and then transferred to either E2 + PTU media or E2 + PTU + Mtz media in 35$\mathrm{mm}$ petri dishes. Embryos were maintained in the dark at $28^{\circ} \mathrm{C}$ for $48 \mathrm{~h}$, and the medium was changed at $24 \mathrm{~h}$. At 5 dpf, the embryos were transferred to E2 + PTU media for $24 \mathrm{~h}$ under normal $12 \mathrm{~h}$ light/12 h dark conditions, fixed in $4 \%$ paraformaldehyde for immunohistochemistry, and subjected to a TUNEL assay (In Situ Cell Death Detection Kit, TMR red, Roche). To examine the stress response, a pool of 30 embryos was used as an experimental unit, and 6 experimental units were tested per treatment. Mtz treatment was performed as above, but the E2 media were not supplemented with PTU.

\section{Stressor treatment and cortisol assay}

Larvae were subjected to a stressor paradigm, which utilized hydrodynamic flow stimulation (HF) using a Variomag Poly 15 stirrer plate (Thermo Scientific) and a plastic-covered stirrer (Magnetic stir bar micro PTFE $6 \mathrm{~mm} \times 3 \mathrm{~mm}$; Fisher Scientific) (Castillo-Ramirez, L., unpublished data). The stimulation was performed for $3 \mathrm{mi}$ nutes using a speed of $330 \mathrm{rpm}$. Samples were collected 10 minutes after onset of stimulation. For collection of samples, ice-cold water was added to the individual petri dishes to immobilize the larvae. They were then transferred to Eppendorf tubes, the remaining medium was removed, and the larvae were frozen in an ethanol/dry ice bath. The samples were stored at $-20^{\circ} \mathrm{C}$. All stimulations and collection of samples were performed between 11:00 and 14:00. The cortisol ELISA assay was performed as previously described [59].

\section{Statistical analysis}

Cortisol data are shown as mean and standard error of the mean (S.E.M.). We used One sample t-tests (against 0 values) and Two way ANOVAs, followed by Bonferroni's post-hoc tests for multiple group comparisons. Analyses were carried out using MS-Excel (Microsoft; Redmond, WA, USA) and Prism 5(Graphpad Software Inc.; San Diego, CA, USA).

\section{Additional files} Additional file 1: Figure S1. ClustalW 2.1 nucleotide alignment output
of the individual ECRs flanking the otp gene in gnathostomes. The table
below each alignment shows the length of the ECR identified in every
species and the pairwise percentage of identity. The TATA box is
underlined in the ECR1 alignment. Hs: Homo sapiens; LC: Latimeria
chalumnae; Dr: Danio rerio; Cm: Challorhincus milii.
Additional file 2: Table S1. Primer sequences used in the present work
to amplify individual ECRs of the otpa gene.

\section{Abbreviations}

Avp: Arginine vasopressin; ACTH: Adrenocorticotropic hormone; Cck: Cholecystokinin; Crh: Corticotropin-releasing hormone; CRM: cis-regulatory module; DA cells: Dopaminergic cells; ECR: Evolutionarily conserved region; FISH: Fluorescent in situ hybridization; GCs: Glucocorticoids; HPA: Hypothalamic-pituitary-adrenal axis; HPI: Hypothalamic-pituitary-interrenal axis; IHC: Immunohistochemistry; Mtz: Metronidazole; NM: Nitroreductasemetronidazole system; NPO: Neurosecretory preoptic area; Otp: Orthopedia transcription factor; Oxt: Oxytocin; Penka: Proenkephalin a; Sst1.1: Somatostatin; Vip: Vasoactive intestinal peptide.

\section{Competing interests}

The authors declare that they have no competing interests.

\section{Authors' contributions}

JAGT and SR conceived and designed the experiments. JAGT, UH, PL and LACR performed the experiments. JAGT, UH, SR, PL and LACR analyzed the data. JAGT, SR and UH wrote the manuscript. All authors read and approved the final manuscript.

\section{Acknowledgements}

We would like to thank the members of Soojin Ryu's lab for critical discussion and contribution to this work, in particular Dr. Rodrigo De Marco and Dr. Andrea Wolf. We also thank Regina Singer, Markus Lutz, Gabi Shoeman and Angelika Schoell for technical assistance and expert fish care. The pZED vector was kindly provided by Dr. Jose Luis Gomez-Skarmetta. We would like to thank Dr. A. F. Parlow, from the Medical Center, University of California, Los Angeles, and the National Hormone and Peptide Program, National Institute of Diabetes and Digestive and Kidney Diseases, for kindly providing the anti-ACTH antibody. This study was funded by the Max Planck Society.

\section{Author details}

'Developmental Genetics of the Nervous System, Max Planck Institute for Medical Research, Jahnstrasse 29, D-69120 Heidelberg, Germany. ${ }^{2}$ Current address: Centre for Organismal Studies (COS), University of Heidelberg, Im Neuenheimer Feld 230, D-69120 Heidelberg, Germany. ${ }^{3}$ The Hartmut Hoffmann-Berling International Graduate School of Molecular and Cellular Biology, University of Heidelberg, Heidelberg, Germany. 
Received: 10 June 2014 Accepted: 11 November 2014 Published online: 27 November 2014

\section{References}

1. Acampora D, Postiglione MP, Avantaggiato V, Di Bonito M, Simeone A: The role of Otx and Otp genes in brain development. Int J Dev Biol 2000, 44(6):669-677.

2. Simeone A, D'Apice MR, Nigro V, Casanova J, Graziani F, Acampora D, Avantaggiato V: Orthopedia, a novel homeobox-containing gene expressed in the developing CNS of both mouse and Drosophila. Neuron 1994, 13(1):83-101

3. Wang W, Lufkin T: The murine Otp homeobox gene plays an essential role in the specification of neuronal cell lineages in the developing hypothalamus. Dev Biol 2000, 227(2):432-449.

4. Acampora D, Postiglione MP, Avantaggiato V, Di Bonito M, Vaccarino FM, Michaud J, Simeone A: Progressive impairment of developing neuroendocrine cell lineages in the hypothalamus of mice lacking the Orthopedia gene. Genes Dev 1999, 13(21):2787-2800.

5. Blechman J, Borodovsky N, Eisenberg M, Nabel-Rosen H, Grimm J, Levkowitz $\mathrm{G}$ : Specification of hypothalamic neurons by dual regulation of the homeodomain protein Orthopedia. Development 2007, 134(24):4417-4426.

6. Eaton JL, Glasgow E: Zebrafish orthopedia (otp) is required for isotocin cell development. Dev Genes Evol 2007, 217(2):149-158.

7. Eaton JL, Holmqvist B, Glasgow E: Ontogeny of vasotocin-expressing cells in zebrafish: selective requirement for the transcriptional regulators orthopedia and single-minded 1 in the preoptic area. Dev Dyn 2008, 237(4):995-1005.

8. Goshu E, Jin H, Lovejoy J, Marion JF, Michaud JL, Fan CM: Sim2 contributes to neuroendocrine hormone gene expression in the anterior hypothalamus. Mol Endocrinol 2004, 18(5):1251-1262.

9. Löhr H, Ryu S, Driever W: Zebrafish diencephalic A11-related dopaminergic neurons share a conserved transcriptional network with neuroendocrine cell lineages. Development 2009, 136(6):1007-1017.

10. Michaud JL, Rosenquist T, May NR, Fan CM: Development of neuroendocrine lineages requires the bHLH-PAS transcription factor SIM1. Genes Dev 1998, 12(20):3264-3275.

11. Ryu S, Mahler J, Acampora D, Holzschuh J, Erhardt S, Omodei D, Simeone A, Driever W: Orthopedia homeodomain protein is essential for diencephalic dopaminergic neuron development. Curr Biol 2007, 17(10):873-880

12. Tessmar-Raible K, Raible F, Christodoulou F, Guy K, Rembold M, Hausen H, Arendt D: Conserved sensory-neurosecretory cell types in annelid and fish forebrain: insights into hypothalamus evolution. Cell 2007, 129(7):1389-1400.

13. Tessmar-Raible K: The evolution of neurosecretory centers in bilaterian forebrains: insights from protostomes. Semin Cell Dev Biol 2007, 18(4):492-501.

14. Norris DO, ebrary Inc: Vertebrate Endocrinology. 4th edition. Amsterdam, Boston: Elsevier Academic Press; 2007.

15. Decatur WA, Hall JA, Smith JJ, Li W, Sower SA: Insight from the lamprey genome: glimpsing early vertebrate development via neuroendocrineassociated genes and shared synteny of gonadotropin-releasing hormone (GnRH). Gen Comp Endocrinol 2013, 192:237-245.

16. Sower SA, Freamat M, Kavanaugh SI: The origins of the vertebrate hypothalamic-pituitary-gonadal (HPG) and hypothalamic-pituitarythyroid (HPT) endocrine systems: new insights from lampreys. Gen Comp Endocrinol 2009, 161(1):20-29.

17. Takahashi A, Kobayashi Y, Mizusawa K: The pituitary-interrenal axis of fish: a review focusing on the lamprey and flounder. Gen Comp Endocrinol 2013, 188:54-59.

18. Charmandari E, Tsigos C, Chrousos G: Endocrinology of the stress response. Annu Rev Physiol 2005, 67:259-284.

19. Wendelaar Bonga SE: The stress response in fish. Physiol Rev 1997, 77(3):591-625

20. Burnstein KL, Cidlowski JA: Regulation of gene expression by glucocorticoids. Annu Rev Physiol 1989, 51:683-699.

21. Leung K, Munck A: Peripheral actions of glucocorticoids. Annu Rev Physio 1975, 37:245-272.

22. Fernandes AM, Beddows E, Filippi A, Driever W: Orthopedia transcription factor otpa and otpb paralogous genes function during dopaminergic and neuroendocrine cell specification in larval zebrafish. PLoS One 2013, 8(9):e75002.

23. Wolf A, Ryu S: Specification of posterior hypothalamic neurons requires coordinated activities of Fezf2, Otp, Sim1a and Foxb1.2. Development 2013, 140(8):1762-1773.
24. Shimogori T, Lee DA, Miranda-Angulo A, Yang Y, Wang H, Jiang L, Yoshida AC, Kataoka A, Mashiko H, Avetisyan M, Qi L, Qian J, Blackshaw S: A genomic atlas of mouse hypothalamic development. Nat Neurosci 2010, 13(6):767-775.

25. Alonso ME, Pernaute B, Crespo M, Gómez-Skarmeta UL, Manzanares M: Understanding the regulatory genome. Int J Dev Biol 2009, 53(8-10):1367-1378.

26. Chatterjee S, Lufkin T: Regulatory genomics: Insights from the zebrafish. Curr Top Genet 2012, 5:1-10.

27. Steenbergen PJ, Richardson MK, Champagne DL: The use of the zebrafish model in stress research. Prog Neuropsychopharmacol Biol Psychiatry 2011, 35(6):1432-1451.

28. Herget U, Wolf A, Wullimann MF, Ryu S: Molecular neuroanatomy and chemoarchitecture of the neurosecretory preoptic-hypothalamic area in zebrafish larvae. J Comp Neurol 2014, 522:1542-1564.

29. Kikuta $H$, Laplante $M$, Navratilova $P$, Komisarczuk $A Z$, Engström PG, Fredman D, Akalin A, Caccamo M, Sealy I, Howe K, Ghislain J, Pezeron G, Mourrain P, Ellingsen S, Oates AC, Thisse C, Thisse B, Foucher I, Adolf B, Geling A, Lenhard B, Becker TS: Genomic regulatory blocks encompass multiple neighboring genes and maintain conserved synteny in vertebrates. Genome Res 2007, 17(5):545-555.

30. Fujimoto E, Stevenson TJ, Chien CB, Bonkowsky JL: Identification of a dopaminergic enhancer indicates complexity in vertebrate dopamine neuron phenotype specification. Dev Biol 2011, 352(2):393-404.

31. Woolfe A, Goodson M, Goode DK, Snell P, McEwen GK, Vavouri T, Smith SF North P, Callaway H, Kelly K, Walter K, Abnizova I, Gilks W, Edwards YJ, Cooke JE, Elgar G: Highly conserved non-coding sequences are associated with vertebrate development. PLoS Biol 2005, 3(1):e7.

32. Frazer KA, Elnitski L, Church DM, Dubchak I, Hardison RC: Cross-species sequence comparisons: a review of methods and available resources. Genome Res 2003, 13(1):1-12.

33. Altschul SF, Gish W, Miller W, Myers EW, Lipman DJ: Basic local alignment search tool. J Mol Biol 1990, 215(3):403-410.

34. Flicek P, Amode MR, Barrell D, Beal K, Billis K, Brent S, Carvalho-Silva D, Clapham P, Coates G, Fitzgerald S, Gil L, Girón CG, Gordon L, Hourlier T, Hunt S, Johnson N, Juettemann T, Kähäri AK, Keenan S, Kulesha E, Martin FJ, Maurel T, McLaren WM, Murphy DN, Nag R, Overduin B, Pignatelli M, Pritchard B, Pritchard E, Riat HS, et al: Ensembl 2014. Nucleic Acids Res 2014, 42(Database issue): D749-D755.

35. Venkatesh B, Lee AP, Ravi V, Maurya AK, Lian MM, Swann JB, Ohta Y, Flajnik MF, Sutoh Y, Kasahara M, Hoon S, Gangu V, Roy SW, Irimia M, Korzh V, Kondrychyn I, Lim ZW, Tay BH, Tohari S, Kong KW, Ho S, Lorente-Galdos B, Quilez J, Marques-Bonet T, Raney BJ, Ingham PW, Tay A, Hillier LW, Minx P, Boehm $T$, et al: Elephant shark genome provides unique insights into gnathostome evolution. Nature 2014, 505(7482):174-179.

36. Mehta TK, Ravi V, Yamasaki S, Lee AP, Lian MM, Tay BH, Tohari S, Yanai S, Tay A, Brenner S, Venkatesh B: Evidence for at least six Hox clusters in the Japanese lamprey (Lethenteron japonicum). Proc Natl Acad Sci U S A 2013, 110(40):16044-16049.

37. Bessa J, Tena JJ, de la Calle-Mustienes E, Fernández-Miñán A, Naranjo S, Fernández A, Montoliu L, Akalin A, Lenhard B, Casares F, Gómez-Skarmeta $J$ L: Zebrafish enhancer detection (ZED) vector: a new tool to facilitate transgenesis and the functional analysis of cis-regulatory regions in zebrafish. Dev Dyn 2009, 238(9):2409-2417.

38. Beretta CA, Dross N, Guiterrez-Triana JA, Ryu S, Carl M: Habenula circuit development: past, present, and future. Front Neurosci 2012, 6:51.

39. Kawakami K, Shima A, Kawakami N: Identification of a functional transposase of the Tol2 element, an Ac-like element from the Japanese medaka fish, and its transposition in the zebrafish germ lineage. Proc Natl Acad Sci U S A 2000, 97(21):11403-11408.

40. Uhlmann T, Boeing S, Lehmbacher M, Meisterernst M: The VP16 activation domain establishes an active mediator lacking CDK8 in vivo. J Bio/ Chem 2007, 282(4):2163-2173.

41. Curado S, Anderson RM, Jungblut B, Mumm J, Schroeter E, Stainier DY: Conditional targeted cell ablation in zebrafish: a new tool for regeneration studies. Dev Dyn 2007, 236(4):1025-1035.

42. Pisharath H, Rhee JM, Swanson MA, Leach SD, Parsons MJ: Targeted ablation of beta cells in the embryonic zebrafish pancreas using E. coli nitroreductase. Mech Dev 2007, 124(3):218-229.

43. Drake JA, Bird C, Nemesh J, Thomas DJ, Newton-Cheh C, Reymond A, Excoffier L, Attar H, Antonarakis SE, Dermitzakis ET, Hirschhorn JN: Conserved noncoding sequences are selectively constrained and not mutation cold spots. Nat Genet 2006, 38(2):223-227. 
44. Harmston N, Baresic A, Lenhard B: The mystery of extreme non-coding conservation. Philos Trans R Soc Lond B Biol Sci 2013, 368(1632):20130021.

45. Nelson AC, Wardle FC: Conserved non-coding elements and cis regulation: actions speak louder than words. Development 2013, 140(7):1385-1395.

46. Smith JJ, Antonacci F, Eichler EE, Amemiya CT: Programmed loss of millions of base pairs from a vertebrate genome. Proc Natl Acad Sci U S A 2009, 106(27):11212-11217.

47. Shimeld SM, Donoghue PC: Evolutionary crossroads in developmental biology: cyclostomes (lamprey and hagfish). Development 2012, 139(12):2091-2099.

48. Spitz F, Furlong EE: Transcription factors: from enhancer binding to developmental control. Nat Rev Genet 2012, 13(9):613-626.

49. Carroll SB: Evo-devo and an expanding evolutionary synthesis: a genetic theory of morphological evolution. Cell 2008, 134(1):25-36.

50. Prud'homme B, Gompel N, Carroll SB: Emerging principles of regulatory evolution. Proc Natl Acad Sci U S A 2007, 104(Suppl 1):8605-8612.

51. Meyer A, Van de Peer Y: From 2R to 3R: evidence for a fish-specific genome duplication (FSGD). Bioessays 2005, 27(9):937-945.

52. Postlethwait J, Amores A, Cresko W, Singer A, Yan YL: Subfunction partitioning, the teleost radiation and the annotation of the human genome. Trends Genet 2004, 20(10):481-490

53. Van de Peer $Y$, Maere $S$, Meyer A: The evolutionary significance of ancient genome duplications. Nat Rev Genet 2009, 10(10):725-732.

54. Alsop D, Vijayan MM: Development of the corticosteroid stress axis and receptor expression in zebrafish. Am J Physiol Regul Integr Comp Physiol 2008, 294(3):R711-R719.

55. Lambert AM, Bonkowsky JL, Masino MA: The conserved dopaminergic diencephalospinal tract mediates vertebrate locomotor development in zebrafish larvae. J Neurosci 2012, 32(39):13488-13500.

56. Westerfield M: The zebrafish book. A guide for the laboratory use of zebrafish (Danio rerio). 4th edition. Eugene: University of Oregon Press; 2000.

57. Siemering KR, Golbik R, Sever R, Haseloff J: Mutations that suppress the thermosensitivity of green fluorescent protein. Curr Biol 1996, 6(12):1653-1663.

58. Sekkali B, Tran HT, Crabbe E, De Beule C, Van Roy F, Vleminckx K: Chicken beta-globin insulator overcomes variegation of transgenes in Xenopus embryos. FASEB J 2008, 22(7):2534-2540.

59. Yeh CM, Glöck M, Ryu S: An optimized whole-body cortisol quantification method for assessing stress levels in larval zebrafish. PLoS One 2013, 8(11):e79406.

doi:10.1186/s12861-014-0041-x

Cite this article as: Gutierrez-Triana et al:: A vertebrate-conserved cis-regulatory module for targeted expression in the main hypothalamic regulatory region for the stress response. BMC Developmental Biology 2014 14:41

\section{Submit your next manuscript to BioMed Central and take full advantage of:}

- Convenient online submission

- Thorough peer review

- No space constraints or color figure charges

- Immediate publication on acceptance

- Inclusion in PubMed, CAS, Scopus and Google Scholar

- Research which is freely available for redistribution 